\title{
Anomalous magnetism in hydrogenated graphene
}

\author{
N. A. García-Martínez, ${ }^{1}$ J. L. Lado, ${ }^{1}$ D. Jacob, ${ }^{2,3,4}$ and J. Fernández-Rossier ${ }^{1,5}$ \\ ${ }^{1}$ QuantaLab, International Iberian Nanotechnology Laboratory (INL), 4715-330 Braga, Portugal \\ ${ }^{2}$ Max-Planck-Institut für Mikrostrukturphysik, Weinberg 2, 06120 Halle, Germany \\ ${ }^{3}$ Nano-Bio Spectroscopy Group, Departamento de Física de Materiales, Universidad del País Vasco, \\ UPV/EHU, Av. Tolosa 72, 20018 San Sebastián, Spain \\ ${ }^{4}$ IKERBASQUE, Basque Foundation for Science, Maria Diaz de Haro 3, 48013 Bilbao, Spain \\ ${ }^{5}$ Departamento de Fisica Aplicada, Universidad de Alicante, San Vicente del Raspeig 03690, Spain
}

(Received 3 April 2017; published 5 July 2017)

\begin{abstract}
We revisit the problem of local moment formation in graphene due to chemisorption of individual atomic hydrogen or other analogous $s p^{3}$ covalent functionalizations. We describe graphene with the single-orbital Hubbard model, so that the $\mathrm{H}$ chemisorption is equivalent to a vacancy in the honeycomb lattice. To circumvent artifacts related to periodic unit cells, we use either huge simulation cells of up to $8 \times 10^{5}$ sites, or an embedding scheme that allows the modeling of a single vacancy in an otherwise pristine infinite honeycomb lattice. We find three results that stress the anomalous nature of the magnetic moment $(m)$ in this system. First, in the noninteracting $(U=0)$ zero-temperature $(T=0)$ case, the $m(B)$ is a continuous smooth curve with divergent susceptibility, different from the stepwise constant function found for single unpaired spins in a gapped system. Second, for $U=0$ and $T>0$, the linear susceptibility follows a power law $\propto T^{-\alpha}$ with an exponent of $\alpha=0.77$ different from the conventional Curie law. For $U>0$, in the mean-field approximation, the integrated moment is smaller than $m=1 \mu_{B}$, in contrast with results using periodic unit cells. These three results highlight the fact that the magnetic response of the local moment induced by $s p^{3}$ functionalizations in graphene is different from that of local moments in gapped systems, for which the magnetic moment is quantized and follows a Curie law, and from Pauli paramagnetism in conductors, for which linear susceptibility can be defined at $T=0$.
\end{abstract}

DOI: 10.1103/PhysRevB.96.024403

\section{INTRODUCTION}

One of the central problems in condensed-matter physics is whether the addition of an impurity atom into an otherwise nonmagnetic crystal results in the formation of local moments [1]. In cases when the host is either a conductor with a welldefined Fermi surface, or an insulator with the Fermi energy inside an energy gap, the problem is well understood. In the former case, the formation of a local moment is controlled by the competition between the addition energies of the impurity levels and their broadening, due to quasiparticle tunneling in and out of the impurity [1,2]. This quantity depends on the density of states of the energy of the localized level. In contrast, when the impurity level lies inside a gap, as it happens for donors in semiconductors, the unpaired electronic spin behaves like a paramagnetic center with $S=1 / 2$ [3].

The hydrogenation of graphene has attracted interest for various reasons. In the dense limit, it leads to the opening of a large band gap [4,5]. In the dilute limit, chemisorbed hydrogen was predicted to create an $S=1 / 2$ local moment [6-11] associated with the formation of an $E=0$, or midgap, state that hosts an unpaired electron. Early on, this led to the proposal of hydrogenated graphene as a magnetic material with spin-dependent transport properties [12-14] apt for spintronics in graphene [15]. Recent scanning tunneling microscope experiments [16] match the computed theoretical density of states as a function of both energy and position, which shows a split resonance close to the Dirac point, providing thereby indirect evidence for local moment formation. It has also been proposed that the small local lattice distortion induced by the $s p^{3}$ hybridization enhances the effect of spin-orbit interaction $[17,18]$. This has been proposed as the explanation for the observation of large spin Hall angles in hydrogenated graphene [19]. Resonant scattering with zero-mode resonance has also been considered as a source of enhanced quasiparticle spin relaxation in graphene [20-22].

Chemisorption of atomic hydrogen in graphene structures entails the formation of a strong covalent bond between the carbon $\pi$ orbital and the hydrogen $s$ orbital. The pair of bonding-antibonding states lies far from the Fermi energy. This picture is valid not only for chemisorption of atomic hydrogen, but for a large variety of other $s p^{3}$ adsorbates [11]. Effectively, the result of this hybridization is to remove both one electron and one state from the $\pi$ cloud, which justifies modeling $s p^{3}$ functionalization with the one orbital tight-binding model with the removal of one atomic site, and one electron in the honeycomb lattice. ${ }^{1}$ The removal of a site in the honeycomb lattice breaks sublattice symmetry and produces a zero-energy state [9,23-26].

In gapped graphene structures, such as graphene with a spin-orbit gap [27], graphene nanoribbons [9], or a planar aromatic hydrocarbon molecule, the formation of an in-gap $E=0$ state due to $s p^{3}$ functionalization leads trivially to a local moment formation with $S=1 / 2$, very much as it happens for acceptors and donors in semiconductors. The zero-energy state is singly occupied by one electron that occupies a bound state. The rest of this paper is devoted to a study of the case of $s p^{3}$ chemisorption in infinite gapless graphene. In that situation, the formation of a local moment is not warranted.

\footnotetext{
${ }^{1}$ This vacancy in the effective model is different from a real vacancy in graphene, which entails the formation of dangling bonds from the $s p^{2}$ orbitals, in addition to the removal of the $\pi$ orbital as well.
} 
Whereas the $E=0$ state appears exactly at the energy where the density of states (DOS) vanishes, a finite DOS due to the Dirac bands is infinitesimally close, and its wave function, described with $\psi \propto \frac{1}{x \pm i y}$, is a quasilocalized non-normalizable state [23]. The DOS of the resonant state diverges at $E=0$ and hence lacks a Lorentzian line shape. This situation is genuinely different from conventional magnetic impurities in metals, which are coupled to a bath with a finite DOS and which show conventional Lorentzian line shapes, and it is also different for the situation in which the resonant state lies inside a proper gap.

Previous works usually addressed this problem by using supercells, where translational symmetry is preserved, hence considering not a single defect but a periodic array of them $[6,8,10,16,28]$. In this case, the bands of such a system will always present a gap that at half-filling results in a quantized $m=$ $1 \mu_{B}$ magnetic moment since all the valence bands would be doubly occupied but only one of the two states in the gap would be occupied. While this approach has been proven very useful in many studies, it does not strictly solve the problem of a single impurity in an otherwise pristine graphene sheet. To tackle this problem, we use two different methodologies. On the one hand, we make a Green's-function description of the defected region embedded into an infinite pristine crystal, which yields an exact description but results in computationally relatively expensive calculations. On the other hand, we use the kernel polynomial method, which allows the calculation of spectral properties for huge systems (800 000 atoms) in a computationally efficient way but has the drawback of lower resolution in energy.

The rest of the paper is organized as follows. Section II is devoted to presenting the methods, i.e., the formalism to treat a single impurity in an infinite system. In Secs. III and IV we study the physical properties of single $s p^{3}$ chemisorption on graphene, including the effect of an external magnetic field with and without temperature. Section $\mathrm{V}$ is devoted to the study of electron-electron interactions, and finally in Sec. VI we summarize our work.

\section{METHODS}

In the following, we describe graphene with a one-orbital tight-binding model. Electron-electron interactions are described within the Hubbard approximation. Thus, the energy scales in the Hamiltonian are the first-neighbor hopping $t$ and the Hubbard on-site repulsion $U$. When an external magnetic field is introduced, it is considered to be an in-plane magnetic field $B$ coupled only to the spin degree of freedom. The effect of $s p^{3}$ hybridization is included by the removal of both a site in the honeycomb lattice and one electron, as discussed above.

We use two different techniques to tackle the problem of a single impurity in pristine graphene. The first one consists in the calculation of the (exact) Green's function for a region close to the defect by means of the Dyson equation, using an embedding method described below. The second one is the kernel polynomial method, which allows the calculation of spectral properties of extremely large systems with minimal computational effort.

\section{A. The embedding technique}

We first present a general method to study single impurities in infinite systems, hereafter referred to as the embedding technique, devised earlier by one of us in a similar context [29]. Since a single defect in an infinite system breaks the translational symmetry, we cannot use a Bloch description. Instead, we describe the system in terms of Green's functions, making use of the Dyson equation. We start by dividing the system into two regions: a central unit cell $A$ containing the defect, and the rest of the system $B$ containing everything else, as depicted in Fig. 1(b). The Hamiltonian of the whole (infinite) system can then be written in terms of the two separated contributions, one arising from each isolated region, $H_{0}$, and the other arising from the coupling between the two regions, $W$ :

$$
H=H_{0}+W=\left(\begin{array}{cc}
H_{A} & 0 \\
0 & H_{B}
\end{array}\right)+\left(\begin{array}{cc}
0 & V_{A B} \\
V_{B A} & 0
\end{array}\right) .
$$

The Green's function corresponding to region $A$ can be written (exactly) as

$$
G_{A}(E)=\frac{1}{E+i \eta-H_{A}-\Sigma_{A B}(E)},
$$

where the embedding self-energy $\Sigma_{A B}$ can be calculated from the Green's function of region $B, g_{B}(E)=\left(E+i \eta-H_{B}\right)^{-1}$, as $\Sigma_{A B}(E)=V_{A B} g_{B}(E) V_{B A}$. For numerical reasons, $\eta$ has to be finite, but we checked that the results do not depend on its exact value. We found that $\eta=0.001$ offers a good combination of precision in energy while keeping the convergence time of the Dyson equation reasonable. In general, the Green's function $g_{B}(E)$ for region $B$ is not straightforward to calculate, as $g_{B}(E)$ describes the Green's function of an
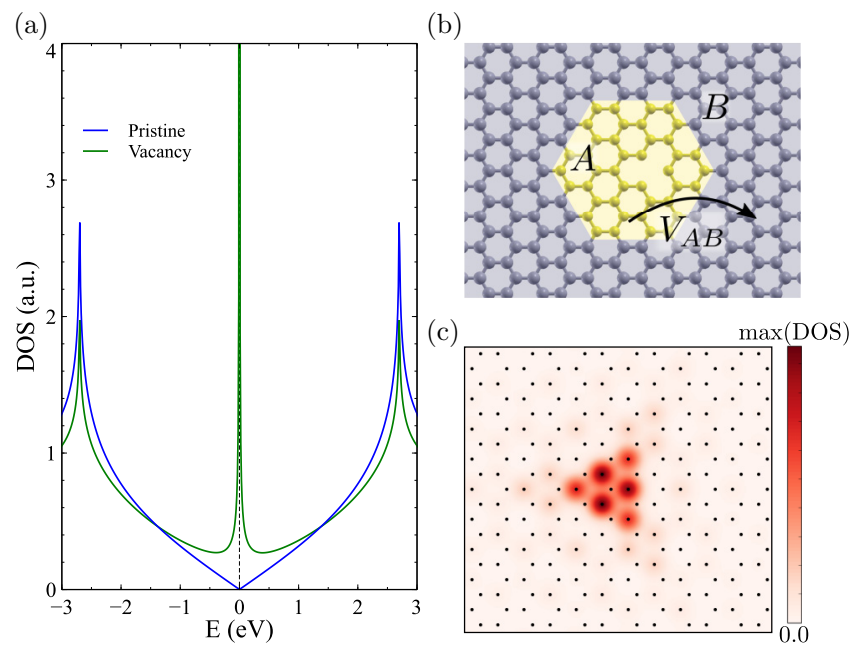

FIG. 1. (a) Total density of states of a single vacancy in an infinite graphene sheet. A divergence in the density of states appears at $E=0$ when the vacancy is introduced. (b) Scheme of the division of the system into a defected unit cell and a pristine environment. (c) Local density of states for the zero-energy state related to a vacancy in graphene. Side by side we can compare the calculations for two unit cells with different geometry. The vacancy is depicted as a white circle. As expected, the spatial distribution of this state is located in the three to six closest atoms to the vacancy. 
infinite system without translational symmetry on account of the missing region $A$. However, the calculation of $\Sigma_{A B}$ is made possible when we consider two facts. First, $\Sigma_{A B}$ does not depend on whatever is in region $A$, and second, Eq. (2) holds true for a pristine system with translational invariance, which permits us to compute $G_{A}(E)$ and evaluate $\Sigma_{A B}=E-H_{A}-\left[G_{A}(E)\right]^{-1}$.

The evaluation of $G_{A}(E)$ is now done by dividing the infinite pristine crystal system into periodic supercells $A^{\prime}$ of the same size and shape as the defect region $A$. The Green's function of region $A^{\prime}$ in the perfect crystal can thus be calculated by integrating the $\vec{k}$-dependent Green's function in the whole Brillouin zone,

$$
G_{A^{\prime}}(E)=\frac{1}{(2 \pi)^{2}} \int_{\mathrm{BZ}}[E+i \eta-H(\vec{k})]^{-1} d^{2} \vec{k}
$$

with $\vec{k}$ the Bloch wave vectors and $H(\vec{k})$ the Bloch Hamiltonian for the pristine host crystal. The final expression for the selfenergy $\Sigma_{A B}$ reads

$$
\begin{aligned}
\Sigma_{A B}(E)= & E+i \eta-H_{A^{\prime}}-\frac{1}{(2 \pi)^{2}} \\
& \times\left(\int_{\mathrm{BZ}}[E+i \eta-H(\vec{k})]^{-1} d^{2} \vec{k}\right)^{-1},
\end{aligned}
$$

where $H_{A^{\prime}}$ describes a region with the same dimensions as the original defective region $A$, but without the $\operatorname{defect}(\mathrm{s})$. This is a general procedure and can be applied for multiband Hamiltonians. As long as the dimensions of the pristine and the defected Hamiltonian are the same, the procedure can deal with more than one defect without computational overhead. Notice that this method does not require the analytic evaluation of the host crystal Green's function necessary in a recently proposed method [30], and it can be applied to a very large class of systems, including superconductors [31].

The combination of Eqs. (2) and (4) allows the computation of the Green's function of the defective area, $G_{A}$, embedded in an otherwise pristine crystal as shown in Fig. 1. The DOS of an atom $i$ in region $A$ can then be calculated from the imaginary part of the Green's function as

$$
\rho_{i}(E)=-\frac{1}{\pi} \operatorname{Im} G_{i, i}(E),
$$

where $G_{i, i}$ is the diagonal matrix element $(i, i)$ of the Green's function. Summing over the contributions from all atoms $i$ in region $A$, the total DOS of region $A$ is obtained.

In Fig. 1 we show the results of the method for the case of a single vacancy in the honeycomb lattice. Figure 1(a) shows the density of states both for pristine graphene, which shows the characteristic $\rho \propto|E|$ around the Dirac point [32], and for the defective case, which presents a diverging zero-energy resonance. The embedding method permits also the calculation of the local density of states as shown in Fig. 1(c), where we show the map of the density of states evaluated at $E=0$, finding that the main contribution for this state comes from the three to six nearest neighbors to the vacancy that belong to the sublattice opposite to that of the missing site [9,23-26]. Of course, for the case of a noninteracting single vacancy, this problem can be dealt with using the standard T matrix theory $[25,27,33]$. The embedding method shows its added value when it is used to treat several vacancies or when interactions are included, as we discuss now.

\section{B. Mean-field Hubbard model}

The Hubbard term acting on every site $i$ reads

$$
H_{U}=U \sum_{i} n_{i, \uparrow} n_{i, \downarrow},
$$

where $n_{i \sigma}$ is the standard number operator for site $i$ with spin $\sigma$. Exact solutions of this model are, in general, not possible so that we use a mean-field approximation:

$$
H_{U} \approx \sum_{i} U\left[\left\langle n_{i, \uparrow}\right\rangle n_{i, \downarrow}+\left\langle n_{i, \downarrow}\right\rangle n_{i, \uparrow}-\left\langle n_{i, \downarrow}\right\rangle\left\langle n_{i, \uparrow}\right\rangle\right],
$$

where $\left\langle n_{i, \sigma}\right\rangle$ stand for the expectation values of the number operators computed with the eigenstates of the mean-field Hamiltonian. Of course, this is a nonlinear problem that is solved self-consistently. Here, this is done in combination with the two-dimensional embedding technique, which is formally similar to the one-dimensional case [34]. In this approach, the occupations in the external region $B$ are frozen to $\left\langle n_{i, \uparrow}\right\rangle=\left\langle n_{i, \downarrow}\right\rangle=\frac{1}{2}$. In contrast, the expected values of the defective cell are calculated by self-consistent iteration.

In a first step, we assume a random guess spin polarization and then compute the expected values of the spin operators $\left\langle n_{i, \sigma}\right\rangle$ by integrating the DOS up to the Fermi energy,

$$
\left\langle n_{i, \sigma}\right\rangle=\int_{-\infty}^{E_{F}} \rho_{\sigma}(E) d E,
$$

which defines a new Hamiltonian for region $A, H_{A} \rightarrow$ $\bar{H}_{A}+H_{U}^{\mathrm{MF}}$, including the mean-field Hubbard term $[9,35]$. Notice that the numerical integration of Eq. (8) is done much more efficiently in the complex plane using Cauchy's integral theorem. Also, it is important to notice that even when the Hamiltonian for region $A$ changes over the self-consistent iterations, the self-energies will not since they do not depend on what is inside of said region. This procedure is iterated until a self-consistent solution is found.

The magnetic moment is calculated as the difference of the expected values of each spin density,

$$
\langle m(i)\rangle \equiv g \mu_{B} \frac{\left\langle n_{i, \uparrow}\right\rangle-\left\langle n_{i, \downarrow}\right\rangle}{2} .
$$

There is a tradeoff between computational cost, due mainly to the size of the region $A$, and the accuracy of the description of the semilocalized nature of the induced magnetism. The role of the chosen size for region $A$ is discussed below.

\section{The kernel polynomial method}

The kernel polynomial method [36] is a spectral method that allows us to calculate spectral properties of very large matrices without explicit diagonalization or inversion of the matrix. This makes the method especially suitable for very large systems described by sparse Hamiltonians, as is the case for the first-neighbor hopping model for the honeycomb lattice considered here. In our case, we set up the Hamiltonian for an extremely large graphene island, with a single vacancy in the center. 
The Chebyshev polynomials form a complete basis in the function space, so that they can be used as a basis to expand any well-behaved function $f(x)$ for $x \in(-1,1)$. The method consists in expanding the density of states in $N$ Chebyshev polynomials $T_{n}(x)$, which are calculated using $T_{0}(x)=1$, $T_{1}(x)=x$, and the recursive relation

$$
T_{n+1}(x)=2 x T_{n}(x)-T_{n-1}(x)
$$

valid for $x \in(-1,1)$.

The first step in the method is to scale the Hamiltonian $H \rightarrow \bar{H}=\sum_{k} \bar{E}_{k}|k\rangle\langle k|$ so that all the eigenstates $\bar{E}_{k}$ fall in the interval $\bar{E}_{k} \in(-1,1)$. The density of states as a function of the scaled energy, at site $i$, is expressed as

$$
\rho_{i}(\bar{E})=\frac{1}{\pi \sqrt{1-\bar{E}^{2}}}\left(\bar{\mu}_{0}+2 \sum_{n=1}^{N-1} \bar{\mu}_{n} T_{n}(\bar{E})\right) .
$$

The coefficients $\bar{\mu}_{n}$ are the modified coefficients of the expansion,

$$
\bar{\mu}_{n}=g_{n}^{N} \mu_{n},
$$

which are obtained using the Jackson kernel [37]

$$
g_{n}^{N}=\frac{(N-n-1) \cos \frac{\pi n}{N+1}+\sin \frac{\pi n}{N+1} \cot \frac{\pi}{N+1}}{N+1},
$$

which improves the convergence of the expansion. The original Chebyshev coefficients are calculated as a conventional functional expansion,

$$
\mu_{n}=\int_{-1}^{1} T_{n}(\bar{E}) \sum_{k} \delta\left(\bar{E}-\bar{E}_{k}\right)|\langle k \mid i\rangle|^{2}=\left\langle i\left|T_{n}(\bar{H})\right| i\right\rangle
$$

with $|i\rangle$ the wave function localized in site $i$. Importantly, the second equality in Eq. (14) relates $\mu_{n}$ to an expression in which the eigenstates $|k\rangle$ of $H$ are absent. Thus, diagonalization of $H$ is not necessary, and the computation of the $\mu_{n}$ coefficients only requires calculating an overlap matrix element involving $T_{n}(H)$. The Chebyshev recursion relation allows us to write down the $\mu_{n}$ coefficients in term of the overlaps with the vectors $\left|\alpha_{n}\right\rangle$,

$$
\mu_{n}=\left\langle\alpha_{0} \mid \alpha_{n}\right\rangle
$$

generated by the recursion relation

$$
\begin{aligned}
\left|\alpha_{0}\right\rangle & =|i\rangle, \\
\left|\alpha_{1}\right\rangle & =\bar{H}\left|\alpha_{0}\right\rangle, \\
\left|\alpha_{n+1}\right\rangle & =2 \bar{H}\left|\alpha_{n}\right\rangle-\left|\alpha_{n-1}\right\rangle .
\end{aligned}
$$

In our case, we will choose a state, $|i\rangle$, localized in the first neighbor of the carbon with the hydrogen adatom. To calculate the previous coefficients we only need matrix vector products, so that the scaling is linear with the size $L$ of the system, in contrast with the $L^{3}$ scaling for exact diagonalization. Our calculations are performed in a graphene island with 800000 atoms, taking an expansion with $N=10000$ polynomials.

\section{NONINTERACTING ZERO-TEMPERATURE MAGNETIZATION}

In this section, we study the spin polarization in the neighborhood of an $s p^{3}$ defect, driven by an external in- plane magnetic field coupled to the electronic spin, at zero temperature and in the noninteracting limit $U=0$. In a gapped graphene system, this problem is straightforward. At $T=0$, the spin density would be dominated by the contribution of the only singly occupied state, the $E=0$ midgap state, whose wave function we denoted by $\psi_{0}(i) \equiv\left\langle i \mid \psi_{0}\right\rangle$. The zero-temperature magnetization in an atom $i$ would be given by

$$
m_{i}(B)=g \mu_{B}\left(\Theta(B)-\frac{1}{2}\right)\left|\psi_{0}(i)\right|^{2}
$$

where $B$ is the magnitude of the magnetic field, $\Theta(B)$ is the step function, $g \simeq 2$ is the gyromagnetic ratio, and $\mu_{B}$ is the Bohr magneton. The local magnetization $m_{i}$ is stepwise constant, and discontinuous at $B=0, m_{i}\left(0^{+}\right)-m_{i}\left(0^{-}\right)=g \mu_{B}\left|\psi_{0}(i)\right|^{2}$. It is apparent that the total moment $M=\sum_{i} m_{i}$ integrates to $M= \pm \frac{g \mu_{B}}{2}$ on account of the normalization of the wave function of the midgap state. This result holds true as long as the Zeeman energy $\mu_{B} B$ is smaller than the gap of the structure.

We now study what happens in the case of infinite pristine graphene, for which there is no gap, and we cannot define a normalized zero-energy state. For that matter, we compute the density of states of the system using the Green's-function embedding approach. This method is trivially adapted to include the Zeeman splitting that introduces a rigid spindependent energy shift $\pm \mu_{B} B$. This symmetric shift allows the expression of all the spectral functions for each of the spin channels in terms of the spinless Green's function, $G^{\sigma}(E)=G\left(E-\sigma \mu_{B} B\right)$, with $\sigma= \pm 1$.

The results for the magnetization of the three first neighbors of an $s p^{3}$ defect in an otherwise pristine graphene are shown in Fig. 2 for a single $s p^{3}$ defect in two scenarios: a gapped finite-size graphene hexagonal island with armchair edges, resulting in the expected stepwise response, and a single defect in otherwise pristine gapless graphene. In both cases, we plot the magnetization of the three atoms closest to the vacancy, $M_{3}=\sum_{i=1}^{3} m_{i}$, which gives the dominant contribution to the defect-induced local moment. The result for the paramagnetic response of a metal is included in Fig. 2 for comparison with a standard case.
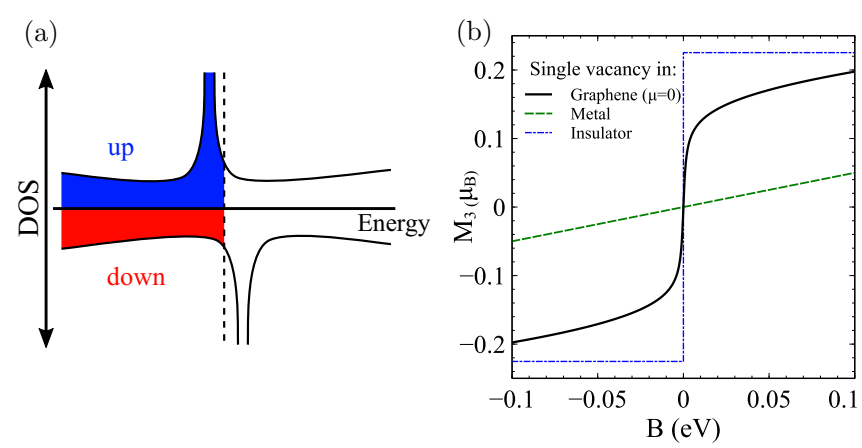

FIG. 2. (a) Sketch for the Zeeman split DOS associated with graphene with an individual $s p^{3}$ functionalization. Panel (b) shows the magnetization of the first three neighbors of the defect as a function of the applied magnetic field for a hydrogen atom in a graphene quantum dot (blue) and a single hydrogen atom in pristine graphene (black). The green line is the result for a conventional metal, modeled by graphene with the chemical potential well above the Dirac point. 
The most prominent feature of the obtained results is the fact that the $M_{3}(B)$ curve is not stepwise constant for the defect in infinite graphene, in marked contrast with the case of the defect in a gapped island. This difference shows the qualitatively different behavior of the zero mode in gapless infinite graphene, compared to the standard case of an in-gap truly localized state. The continuous variation of the magnetic moment can be related to the fact that the zero mode has an intrinsic linewidth that reflects the lack of a gap to host a true localized state.

\section{FINITE-TEMPERATURE SUSCEPTIBILITY}

We now discuss the effect of temperature on the noninteracting $m(B)$ curve. The only effect of temperature is to smear out the occupation of the one-particle levels, so that the expected value of the local magnetization must now include excited states.

To calculate the magnetization in a site $i$ as a function of the magnetic field and the temperature, we just need to compute the difference in the occupation of the spin-up and spin-down density of states weighted with the Fermi-Dirac distribution function. Thus, the local magnetic moment is given by $m_{i}=g \mu_{B}\left\langle s_{z}(i)\right\rangle$ with

$$
\left\langle s_{z}(i)\right\rangle=\frac{1}{2} \int_{-\infty}^{\infty}\left[\rho_{i \uparrow}(E)-\rho_{i \downarrow}(E)\right] f(E, T) d E,
$$

where $f(E, T)$ is the Fermi-Dirac distribution and $\rho_{i \sigma}(E)$ is the spin-resolved density of states. The resulting magnetization of the three closest atoms, $M_{3}$, is shown in Fig. 3(a), computed with two different approaches: the embedding method for a single $s p^{3}$ defect in infinite graphene, and the kernel polynomial method for a single defect on a finite-size island with a very large number of atoms. It is apparent that both methods yield identical results in the chosen range of temperatures.

To highlight the anomalous behavior of the magnetic moment associated with an individual $s p^{3}$ defect, we focus on the spin susceptibility, defined as

$$
\chi(T)=\left.\frac{\partial m(T)}{\partial B}\right|_{B=0} .
$$

For $T=0$, the results of the previous section show that this quantity diverges, both for the gapped and gapless cases. Here we study the dependence of $\chi(T)$ as a function of temperature $T$. For a conventional local moment, the zero-field susceptibility follows the Curie law $\chi(T) \propto T^{-1}$. This result holds true, based on very general considerations, for any spin governed by the Hamiltonian $g \mu_{B} \vec{S} \cdot \vec{B}$ as well as any classical magnetic moment $\vec{M}$ governed by the interaction energy $-\vec{M} \cdot \vec{B}$. In particular, the Curie susceptibility of a single electron in an in-gap level will follow a Curie law.

Numerical derivation of the results of Fig. 3(a) allows for the calculation of $\chi(T)$, shown in Fig. 3(b) in a LogLog representation. It is apparent that the spin susceptibility for the $s p^{3}$ defect on graphene does not follow the Curie law. In particular, we obtain a high-temperature power-law dependence $\chi \propto T^{-\alpha}$, with $\alpha \sim 0.77$, in comparison with the conventional $\alpha=1$. This exponent reflects again the anomalous nature of the $s p^{3}$ local moment in infinite graphene,
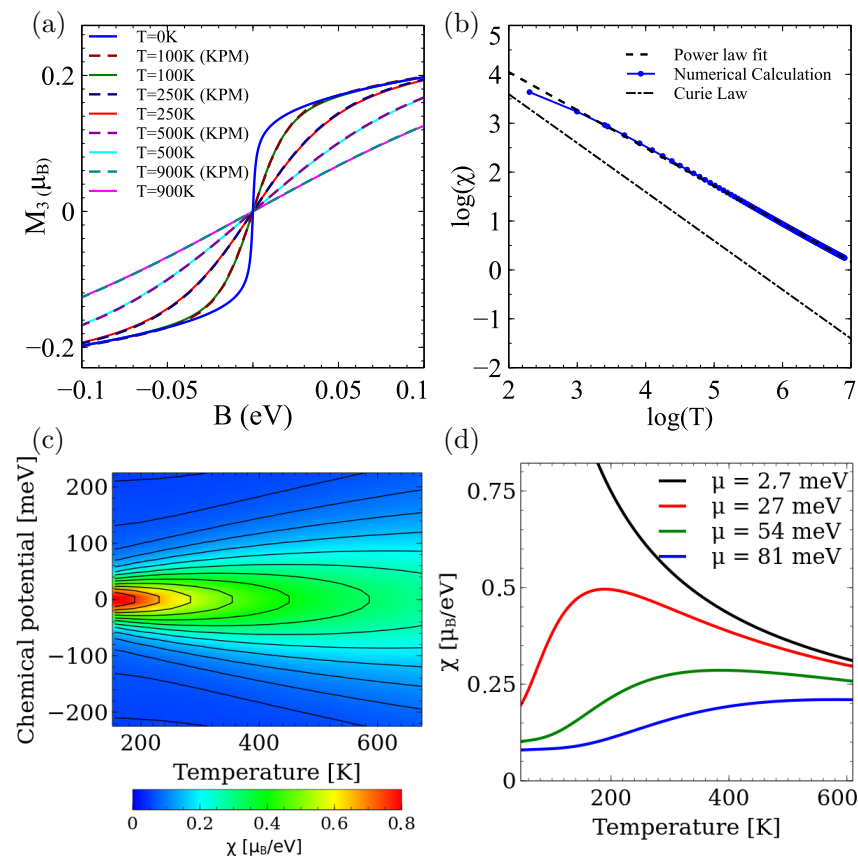

FIG. 3. (a) Magnetization of the first three neighbors of the vacancy as a function of the applied magnetic field for different temperatures. Dashed lines are calculated using the KPM, and continuous lines are calculated using the embedding method. (b) Temperature dependence of the susceptibility in comparison with Curie's law. (c),(d) Dependence of the susceptibility with the doping and the temperature, showing that for some values of $\mu$ there is a nonmonotonous behavior of the susceptibility with temperature.

in marked contrast with the behavior of the same chemical functionalization in a gapped graphene structure. Interestingly, $\chi(T)$ has been measured [38] for defective graphene obtaining a Curie law dependence, probably because the samples used are in fact nanoflakes with small confinement gaps that permit the existence of in-gap states with quantized spins.

Further insight into the magnetic properties of this system is obtained by considering the dependence of the magnetic susceptibility as a function of the graphene chemical potential, which could be modified by gating doping [39], as shown in Figs. 3(c) and 3(d). The maximal local susceptibility is obtained at half-filling and small temperatures, where it decreases monotonically with both temperature and doping. In comparison, in the case of slightly doped samples, the magnetic susceptibility can either increase or decrease as a function of the temperature, showing a maximum at a dopingdependent temperature. The previous behavior can be understood as a crossover from the impurity in an insulator to the metallic regime. Importantly, the local maximum implies that graphene doping introduces an energy scale that determines the temperature for the impurity-metal crossover. In the case of heavily doped samples $(\mu=81 \mathrm{meV})$, the susceptibility grows monotonically with temperature, signaling the conventional metallic regime.

\section{EFFECT OF INTERACTIONS}

We now study the effect of electron-electron interactions in the formation of local magnetic moments associated with 
the $s p^{3}$ functionalization. A single unpaired electron in an in-gap energy level has a spin $S=1 / 2$ (the equivalent of a magnetic moment $\left.m=1 \mu_{B}\right)$. In the current study, this is not the case. The $E=0$ resonance is embedded in a region with a finite DOS (except in just one point, the Dirac energy), and the existence of an emergent magnetic moment follows the Stoner criterion $U \rho\left(E_{F}\right)=1$. For graphene with a single $s p^{3}$ defect, the diverging nature of $\rho(E)$ at $E=0$ signals that the existance of arbitrarily small interactions will give rise to a local magnetic moment. However, because of the coupling of the midgap state to a continuum of states (the linear bands of graphene), it is not obvious a priori whether the local moment should have a quantized $S=1 / 2$ spin. In the following we address this issue, using the mean-field approximation for the Hubbard model and the embedding technique as discussed in previous sections. The Hubbard model, while certainly not a complete description of such a system, offers a simple model with which to gain insight into the possible magnetic solutions for the systems.

\section{A. Local magnetic moment}

In general, the results of the mean-field calculation yield nonzero magnetization above rather small values of $U$. However, the integrated local moment $M=\sum_{i \in A} m_{i}$ is far below the quantized value of $M=1 \mu_{B}$. A characteristic snapshot of the magnetization density computed for a simulation cell with 162 atoms within the mean-field Hubbard model is shown in Fig. 4(a). The dominant magnetic moments appear in the sublattice opposite to that of the defect, but small contributions of opposite sign appear in the same sublattice.

The influence of the coupling to infinite graphene is neatly shown in a calculation where we artificially tune the intensity of the interaction between the central simulation cell $A$ and the rest of graphene. For that matter, we define the following modified full Green's function:

$$
\widetilde{G}_{A}^{\lambda}(E)=\frac{1}{E+i \eta-\widetilde{H}_{A}-\lambda_{\Sigma} \Sigma_{A B}(E)},
$$

where $\lambda_{\Sigma} \in[0,1]$ is a control parameter that smoothly interpolates between the limit where the region $A$ is decoupled $\left(\lambda_{\Sigma}=0\right)$ from the rest of the universe, the quantum dot regime, and the infinite-crystal regime $\left(\lambda_{\Sigma}=1\right)$.

As is shown in Fig. 4(b), in the quantum dot regime $\lambda_{\Sigma}=0$ the magnetic moment is quantized, $M=1 \mu_{B}$. However, as soon as the unit cell is coupled to the rest of the graphene, $\lambda_{\Sigma} \neq 0$, the magnetic moment rapidly becomes nonquantized, with an evolution that depends on the size and geometry of the region $A$.

As soon as $U$ is larger than a small critical $U \simeq 0.01 t$, magnetic solutions are obtained and we find that the noninteger nature of the magnetic moment holds for a wide regime of electronic interactions $U$. The net magnetic moment is an increasing function of $U$ as well as the size of the central region, $N_{C}$ [Figs. 4(c) and 4(d)]. Even for the largest simulation cells, with up to 288 sites, the total magnetic moment remains clearly below $1 \mu_{B}$. However, a representation of the total moment as a function of $N_{C}^{-1}$, not shown, makes it hard to predict whether the extrapolation to an infinite cell would recover the quantized value. Whereas it might be that the (a)
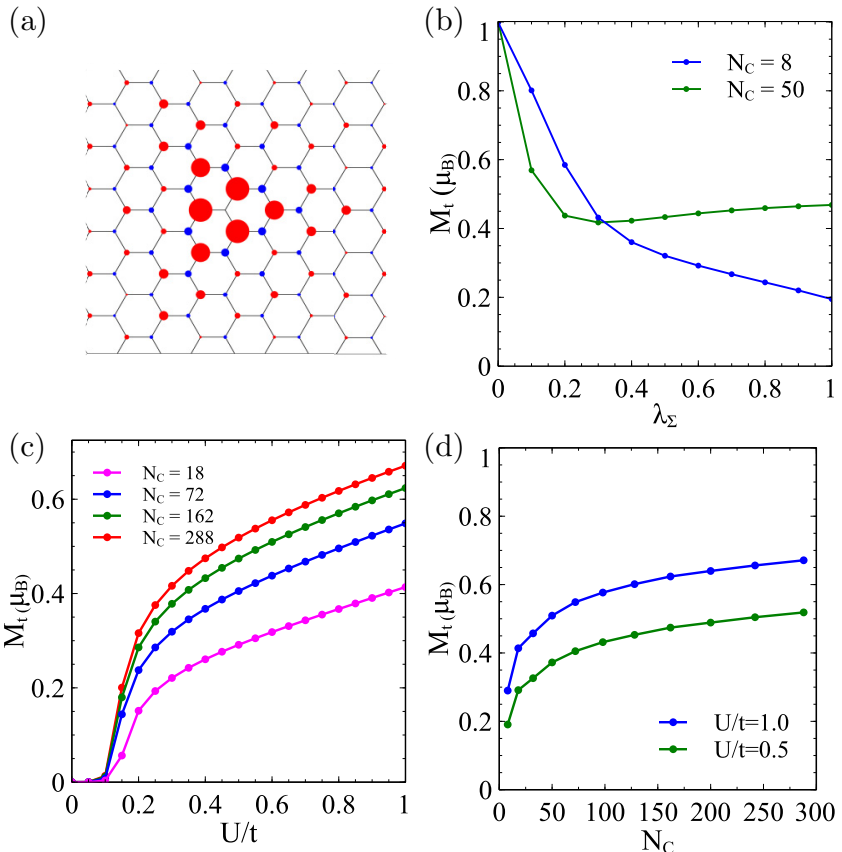

FIG. 4. (a) Magnetization of an individual $s p^{3}$ functionalized system, calculated within the mean-field Hubbard approximation. (b) Total magnetization of the defected region as a function of its coupling to the rest of the otherwise pristine system. (c) Total magnetization of the defected region as a function of the Hubbard $U$ for different sizes of the unit cells. (d) Total magnetization as a function of the size of the unit cell for two $U$ values; notice that the magnetization is far from the expected $m=1 \mu_{B}$ value.

magnetic moment is quantized, our calculations emphasize the rather extended nature of this object.

\section{B. Spin splitting}

Our magnetic self-consistent solutions spontaneously break symmetry and result in a spin-split density of states, as shown in Fig. 5. Importantly, the interacting DOS does not have any integrable singularity, as happens for the $U=0$ case at $E=0$. Summing over spin projections, the total density of states still shows electron-hole symmetry. However, the spin-resolved DOS is split, so for one spin projection the resonance is below
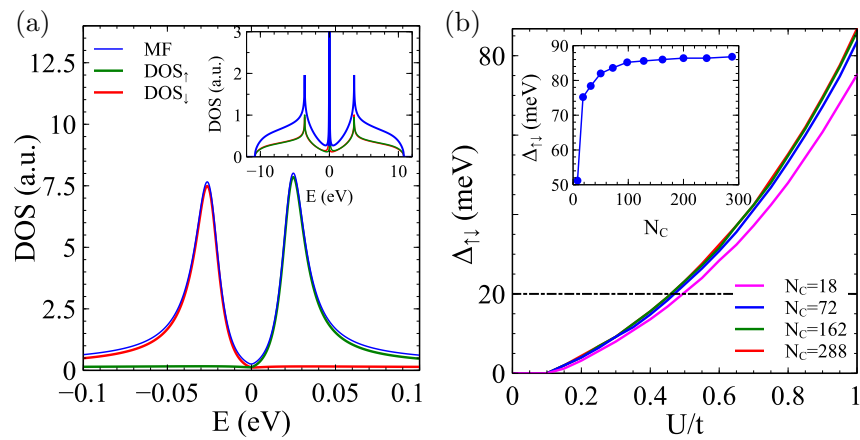

FIG. 5. (a) Total and spin-resolved DOS for the Hubbard model. (b) Spin splitting, $\Delta$, as a function of the Hubbard interaction $U$ and, in the inset, as a function of the size of the unit cell. 
the Fermi energy while for the other it is above, which accounts for the net magnetization. We define the spin splitting $\Delta$ as the difference in energy between these two resonances. We find that $\Delta$ has a superlinear dependence on the Hubbard interaction $U$, as shown in Fig. 5(b). This reflects the fact that the spin splitting is linear both in $U$ and in the magnetic density $m$, which is also an increasing function of $U$. These results depend weakly on the size of the defective region, $A$, in the embedding calculation [inset of Fig. 5(b)].

We now address the connection between the DOS in Fig. 5(a), which shows two spin-split peaks around the Fermi energy, and the STM $d I / d V$ spectra reported by González et al. [16]. Whereas the experimental $d I / d V$ curve also shows two peaks, the interpretation of the experimental observations [16] requires some caution. The spin-split peaks in the meanfield calculation arise from the breaking of spin symmetry. This band splitting does indeed occur in ferromagnetic systems large enough to keep the magnetization frozen along a given direction that defines a spin-quantization axis that permits the definition of the spin orientation of the spin-split bands. The quantum fluctuations of this mean-field picture can be safely neglected for a large enough magnetic moment, but this is definitely not the case of a system that, at most, has $S=1 / 2$.

Therefore, the origin of the two peaks observed in the experiments [16] is not the spin splitting of the graphene energy levels, in line with the discussion of Gonzalez et al. [16]. A more correct interpretation of the split peaks is the following. The STM $d I / d V$ is proportional to the spectral function of the surface, in this case graphene. The spectral function of a localized level has two peaks: one peak, at $E>E_{f}$, is associated with the addition and the other, at $E<E_{f}$, is associated with the removal of an electron in the system. Their energy difference is a measurement of the addition energy of the system, i.e., the Coulomb repulsion between the host localized electron and a second additional electron injected in the system. When the addition energy is larger than the temperature, as in the experiment, the system is in the so-called Coulomb-blockade regime. This entails the existence of an unpaired spin, very much like in the Anderson model [1], and it does not preclude the emergence of the Kondo effect at very low temperatures. The proper treatment of the addition energies in this system would involve solving the single impurity problem of a resonance in a Dirac bath in a many-body framework [28,40,41], which is beyond the scope of the present work.

Finally, for a truly localized level with wave function $\phi_{0}$, described with the Hubbard model, both the addition energy and the mean-field spin splitting are roughly given by $U \sum_{i}\left|\psi_{0}(i)\right|^{4}$. Therefore, even if the spin splitting of the mean-field theory is conceptually different from the addition energy observed experimentally, these two quantities are described roughly by the same formula.

\section{Localization}

Our calculations show that the magnetic moment associated with a hydrogen adatom is delocalized in more than 250 carbon atoms. In this sense, our calculations highlight the anomalous nature of the resonance due to a single $s p^{3}$ impurity in contrast to the phenomenology in gapped systems. This behavior arises from the special condition of the DOS (null only in exactly one point) and the absence of an energy scale that can confine the $E=0$ resonance.

It is worth noting that in real graphene, there are some effects not captured by the first-neighbor Hubbard model that can play a relevant role. First, the existence of second-neighbor carbon hopping breaks electron-hole symmetry and shifts the vacancy state away from $E=0$. Second, single hydrogenation introduces an effective on-site energy in the carbon atom that is actually finite, although rather large, which also leads to a displacement of the resonance away from zero energy. Third, nonlocal electronic interaction in graphene may have a sizable effect on the magnetic moment. And finally, spin-orbit coupling would open a gap of around $0.03 \mathrm{meV}$ [42]. Whether any of the previous perturbations would be capable of moving the system to the conventional quantum dot regime would require a careful study with a first-principles Hamiltonian, which is beyond the scope of the present work.

\section{CONCLUSIONS}

We have addressed the problem of the local moment formation induced by an individual $s p^{3}$ functionalization in graphene, with chemisorbed atomic hydrogen as the main motivation. We model this within the single-orbital Hubbard model, so that the functionalization is modeled as a vacancy in the honeycomb lattice. We have shown that the magnetic moment in this system departs from the conventionally accepted $m=1 \mu_{B}$ picture. This relates to the fact that the lack of a gap in graphene prevents the existence of a standard in-gap state that can host an unpaired electron. Our calculations show that the local moment induced by $s p^{3}$ functionalization in otherwise gapless and pristine graphene gives rise to specific signatures in the magnetic response of the system, such as a non-Curie temperature dependence and a nonlinear (and nonmonotonic for some doping values) magnetic susceptibility. We have also shown by means of mean-field calculations that the resulting magnetic moment is nonquantized in the whole regime explored. Our results should pave the way for future work treating many-body spin fluctuations beyond mean-field theory $[28,40,41]$.

\section{ACKNOWLEDGMENTS}

The authors acknowledge financial support by MarieCurie-ITN 607904-SPINOGRAPH. J.F.-R. acknowledges financial supported by MEC-Spain (Grants No. FIS2013-47328C2-2-P and No. MAT2016-78625-C2) and Generalitat Valenciana (Grant No. ACOMP/2010/070), Prometeo, by European Regional Development Fund (ERDF) funds through the Portuguese Operational Program for Competitiveness and Internationalization COMPETE 2020, and National Funds through FCT, under the Project No. PTDC/FIS-NAN/4662/2014 (016656) [Portuguese Foundation for Science and Technology]. This work has been financially supported in part by FEDER funds. J.L.L. and N.A.G.-M. gratefully acknowledge the hospitality of the Departamento de Fisica Aplicada at the Universidad de Alicante. J.L.L. thanks P. San-Jose for showing the kernel polynomial method. We acknowledge Ivan Brihuega, Manuel Melle-Franco, Juanjo Palacios, and H. Gonzalez for fruitful discussions. 
[1] P. W. Anderson, Phys. Rev. 124, 41 (1961).

[2] M. D. Daybell and W. A. Steyert, Rev. Mod. Phys. 40, 380 (1968).

[3] C. P. Slichter, Phys. Rev. 99, 479 (1955).

[4] D. C. Elias, R. R. Nair, T. M. G. Mohiuddin, S. V. Morozov, P. Blake, M. P. Halsall, A. C. Ferrari, D. W. Boukhvalov, M. I. Katsnelson, A. K. Geim, and K. S. Novoselov, Science 323, 610 (2009).

[5] J. O. Sofo, A. S. Chaudhari, and G. D. Barber, Phys. Rev. B 75, 153401 (2007).

[6] E. J. Duplock, M. Scheffler, and P. J. D. Lindan, Phys. Rev. Lett. 92, 225502 (2004).

[7] O. V. Yazyev and L. Helm, Phys. Rev. B 75, 125408 (2007).

[8] D. W. Boukhvalov, M. I. Katsnelson, and A. I. Lichtenstein, Phys. Rev. B 77, 035427 (2008).

[9] J. J. Palacios, J. Fernández-Rossier, and L. Brey, Phys. Rev. B 77, 195428 (2008).

[10] O. V. Yazyev, Rep. Prog. Phys. 73, 056501 (2010).

[11] E. J. Santos, A. Ayuela, and D. Sánchez-Portal, New J. Phys. 14, 043022 (2012).

[12] D. Soriano, F. Muñoz-Rojas, J. Fernández-Rossier, and J. J. Palacios, Phys. Rev. B 81, 165409 (2010).

[13] N. Leconte, D. Soriano, S. Roche, P. Ordejon, J.-C. Charlier, and J. J. Palacios, ACS Nano 5, 3987 (2011).

[14] D. Soriano, N. Leconte, P. Ordejón, J.-C. Charlier, J.-J. Palacios, and S. Roche, Phys. Rev. Lett. 107, 016602 (2011).

[15] W. Han, R. K. Kawakami, M. Gmitra, and J. Fabian, Nat. Nanotechnol. 9, 794 (2014).

[16] H. González-Herrero, J. M. Gómez-Rodríguez, P. Mallet, M. Moaied, J. J. Palacios, C. Salgado, M. M. Ugeda, J.-Y. Veuillen, F. Yndurain, and I. Brihuega, Science 352, 437 (2016).

[17] A. H. C. Neto and F. Guinea, Phys. Rev. Lett. 103, 026804 (2009).

[18] M. Gmitra, D. Kochan, and J. Fabian, Phys. Rev. Lett. 110, 246602 (2013).

[19] J. Balakrishnan, G. K. W. Koon, M. Jaiswal, A. C. Neto, and B. Özyilmaz, Nat. Phys. 9, 284 (2013).

[20] M. Wojtaszek, I. J. Vera-Marun, T. Maassen, and B. J. van Wees, Phys. Rev. B 87, 081402 (2013).

[21] D. Kochan, M. Gmitra, and J. Fabian, Phys. Rev. Lett. 112, 116602 (2014).
[22] D. Soriano, D. V. Tuan, S. M. Dubois, M. Gmitra, A. W. Cummings, D. Kochan, F. Ortmann, J.-C. Charlier, J. Fabian, and S. Roche, 2D Mater. 2, 022002 (2015).

[23] V. M. Pereira, F. Guinea, J. M. B. L. dos Santos, N. M. R. Peres, and A. H. C. Neto, Phys. Rev. Lett. 96, 036801 (2006).

[24] H. Kumazaki and D. S. Hirashima, J. Phys. Soc. Jpn. 76, 034707 (2007).

[25] T. O. Wehling, A. V. Balatsky, M. I. Katsnelson, A. I. Lichtenstein, K. Scharnberg, and R. Wiesendanger, Phys. Rev. B 75, 125425 (2007).

[26] V. M. Pereira, J. M. B. L. dos Santos, and A. H. C. Neto, Phys. Rev. B 77, 115109 (2008).

[27] J. W. González and J. Fernández-Rossier, Phys. Rev. B 86, 115327 (2012).

[28] J. O. Sofo, G. Usaj, P. S. Cornaglia, A. M. Suarez, A. D. Hernández-Nieves, and C. A. Balseiro, Phys. Rev. B 85, 115405 (2012).

[29] D. Jacob and G. Kotliar, Phys. Rev. B 82, 085423 (2010).

[30] M. Settnes, S. R. Power, J. Lin, D. H. Petersen, and A.-P. Jauho, Phys. Rev. B 91, 125408 (2015).

[31] J. Lado and J. Fernández-Rossier, 2D Mater. 3, 025001 (2016).

[32] M. I. Katsnelson, Graphene: Carbon in Two Dimensions (Cambridge University Press, Cambridge, 2012).

[33] E. N. Economou, Green's Functions in Quantum Physics, Vol. 7 (Springer, Berlin, Heidelberg, 2006).

[34] F. Muñoz-Rojas, J. Fernández-Rossier, and J. J. Palacios, Phys. Rev. Lett. 102, 136810 (2009).

[35] J. Fernández-Rossier and J. J. Palacios, Phys. Rev. Lett. 99, 177204 (2007).

[36] A. Weiße, G. Wellein, A. Alvermann, and H. Fehske, Rev. Mod. Phys. 78, 275 (2006).

[37] D. Jackson, Trans. Am. Math. Soc. 13, 491 (1912).

[38] R. R. Nair, M. Sepioni, I.-L. Tsai, O. Lehtinen, J. Keinonen, A. V. Krasheninnikov, T. Thomson, A. K. Geim, and I. V. Grigorieva, Nat. Phys. 8, 199 (2012).

[39] R. R. Nair, I. -L. Tsai, M. Sepioni, O. Lehtinen, J. Keinonen, A. V. Krasheninnikov, A. H. C. Neto, M. I. Katsnelson, A. K. Geim, and I. V. Grigorieva, Nat. Commun. 4, 2010 (2013).

[40] P. Haase, S. Fuchs, T. Pruschke, H. Ochoa, and F. Guinea, Phys. Rev. B 83, 241408 (2011).

[41] A. K. Mitchell and L. Fritz, Phys. Rev. B 88, 075104 (2013).

[42] M. Gmitra, S. Konschuh, C. Ertler, C. Ambrosch-Draxl, and J. Fabian, Phys. Rev. B 80, 235431 (2009). 